\title{
Protein Biomarkers in Uveitis
}

\section{OPEN ACCESS}

Edited by:

Peizeng Yang,

First Affiliated Hospital of Chongqing

Medical University, China

Reviewed by:

Krishna Ramachandra Murthy,

Vittala International Institute of

Ophthalmology, India

Yohei Tomita,

Boston Children's Hospital and

Harvard Medical School, United States

*Correspondence:

Reema Bansal

drreemab@rediffmail.com

Specialty section:

This article was submitted to

Autoimmune and

Autoinflammatory Disorders,

a section of the journal

Frontiers in Immunology

Received: 25 September 2020

Accepted: 02 November 2020

Published: 03 December 2020

Citation:

Bansal $R$ and Gupta A (2020) Protein

Biomarkers in Uveitis.

Front. Immunol. 11:610428.

doi: 10.3389/fimmu.2020.610428

\section{Reema Bansal * and Amod Gupta \\ Advanced Eye Centre, Post Graduate Institute of Medical Education and Research, Chandigarh, India}

The diseases affecting the retina or uvea (iris, ciliary body, or choroid) generate changes in the biochemical or protein composition of ocular fluids/tissues due to disruption of bloodretinal barrier. Ocular infections and inflammations are sight-threatening diseases associated with various infectious and non-infectious etiologies. Several etiological entities cause uveitis, a complex intraocular inflammatory disease. These causes of uveitis differ in different populations due to geographical, racial, and socioeconomic variations. While clinical appearance is sufficiently diagnostic in many diseases, some of the uveitic entities manifest nonspecific or atypical clinical presentation. Identification of biomarkers in such diseases is an important aid in their diagnostic armamentarium. Different diseases and their different severity states release varying concentrations of proteins, which can serve as biomarkers. Proteomics is a high throughput technology and a powerful screening tool for serum biomarkers in various diseases that identifies proteins by mass spectrometry and helps to improve the understanding of pathogenesis of a disease. Proteins determine the biological state of a cell. Once identified as biomarkers, they serve as future diagnostic and pharmaceutical targets. With a potential to redirect the diagnosis of idiopathic uveitis, ocular proteomics provide a new insight into the pathophysiology and therapeutics of various ocular inflammatory diseases. Tears, aqueous and vitreous humor represent potential repositories for proteomic biomarkers discovery in uveitis. With an extensive proteomics work done on animal models of uveitis, various types of human uveitis are being subjected to proteome analysis for biomarker discovery in different ocular fluids (vitreous, aqueous, or tears). 


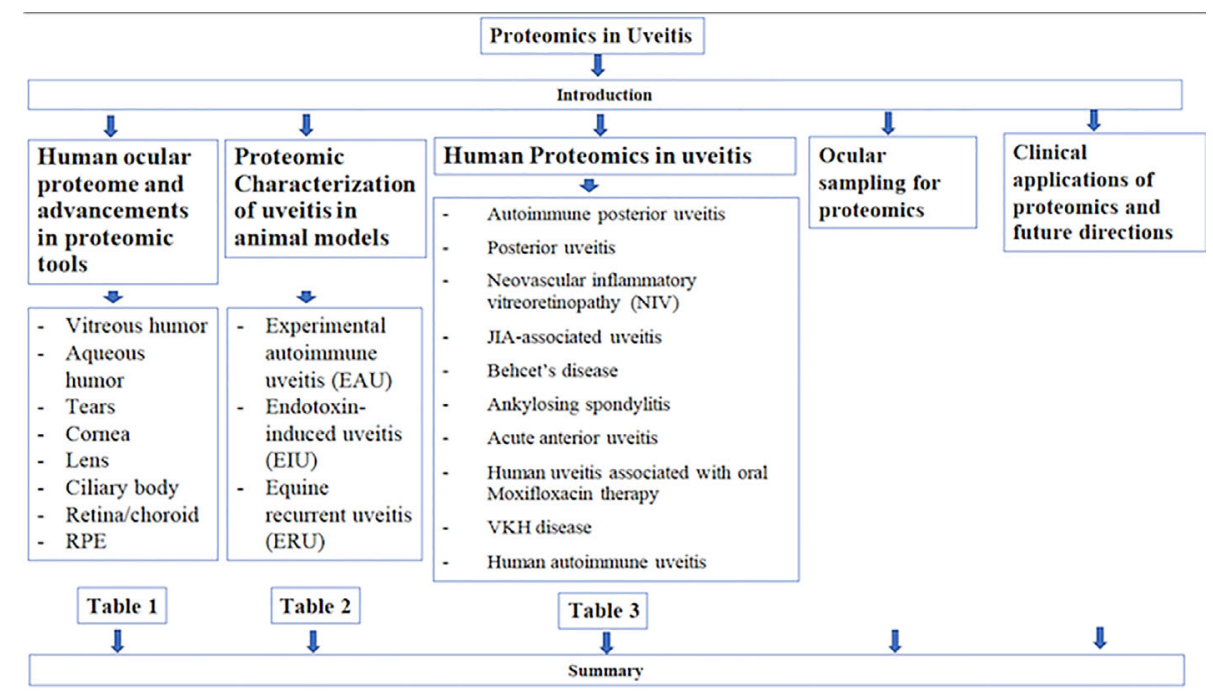

GRAPHICAL ABSTRACT | Flowchart of article outline.

\section{INTRODUCTION}

Different diseases and their different severity states release varying concentrations of proteins, which can serve as biomarkers. Proteomics is a high throughput technology and a powerful screening tool for serum biomarkers in various diseases that identifies proteins by mass spectrometry and helps to improve the understanding of pathogenesis of a disease. Proteins determine the biological state of a cell. Once identified as biomarkers, they serve as future diagnostic and pharmaceutical targets. While serum, plasma, urine, or sputum samples have been utilized for identifying disease biomarkers, vitreous proteomics is emerging rapidly in ocular diseases (1-3).

Ocular proteomics is a promising field aimed at the advancement of diagnostics and therapeutics of many debilitating ocular diseases (4-6). The enormous protein profile of the intraocular structures, including the intraocular fluids (vitreous and aqueous humor), forms the basis of research into ocular proteomics. Several attempts have been made to characterize the proteome of the vitreous, both in animal models and humans, including healthy and diseased states. Insights into various aspects of an ocular disease have been provided by identification of individual proteins and networks of functionally related proteins with signaling pathways. As a component of the Biology/Diseasedriven Human Proteome Project (B/D-HPP), which supports the broad application of state-of-the-art measurements of proteins/ proteomes in human diseases, the Human Eye Proteome Project (HEPP) was officially initiated in 2012 to facilitate proteomic studies of the eye $(7,8)$.

The diseases affecting the retina or uvea (iris, ciliary body, or choroid) generate changes in the biochemical or protein composition of ocular fluids/tissues due to disruption of bloodretinal barrier. Ocular infections and inflammations are sightthreatening diseases associated with various infectious and non- infectious etiologies. Intraocular inflammations (infectious or non-infectious) account for $5 \%-20 \%$ of blindness in the developed world and $25 \%$ in the developing world $(9,10)$. Several etiological entities cause uveitis, a complex intraocular inflammatory disease. These causes of uveitis differ in different populations due to geographical, racial, and socioeconomic variations (10). While clinical appearance is sufficiently diagnostic in many diseases, some of the uveitic entities manifest nonspecific or atypical clinical presentation. Identification of biomarkers in such diseases is an important aid in their diagnostic armamentarium. This review highlights the developments and advances in proteomics of intraocular inflammation or uveitis.

\section{HUMAN OCULAR PROTEOME AND ADVANCEMENTS IN PROTEOMIC TOOLS}

\section{Vitreous Humor}

Proteomic analysis of human vitreous humor has revealed a large profile of intracellular and extracellular proteins (11-15). Nourishment of the vitreous humor from the blood vessels of the retina (and ciliary body) and a close proximity to the retina induces several biochemical changes on protein dynamics of the vitreous, which can be detected by a comprehensive proteomic profiling. A number of vitreous fluid proteins have been linked to the etiology of ocular and vitreo-retinal disorders $(16,17)$. The main components of proteomic studies involve separation of proteins from complex mixtures (fractionation), and their identification by mass spectrometry and data analysis. Proteomic methodologies have evolved from gel electrophoresis (in-gel digestion of proteins) to shotgun proteomics (in-solution digestion of proteins) $(18,19)$. The diversity present in the ocular cells, tissues, and fluids warrants an 
adequate sample preparation to produce reliable results from ocular proteomics (20).

Using sodium dodecyl sulphate-polyacrylamide gel electrophoresis (SDS-PAGE), Gao et al. employed liquid chromatography mass spectrometry (LC-MS/MS) and identified 252 proteins in the vitreous of non-diabetic controls (21). Using different prefractionation techniques like onedimensional (1D) SDS-PAGE or liquid-phase isoelectric focusing (IEF), Aretz et al. identified 1111 proteins (12). The presence of 262 unique proteins in all three patient samples probably represented the constitutive protein pattern of human vitreous. They found striking differences between the vitreous and plasma proteome. Only $27 \%$ of their 1111 vitreous proteins were listed as plasma proteins. Contrary to the assumption that most vitreous proteins originate from the plasma, they suggested that the crystalline lens, retina and retinal pigment epithelium (RPE) contribute to the vitreous proteome. The authors also suggested similarities between aqueous and vitreous proteome due to the continuous exchange between the two body fluids.

Presence of high abundant proteins in the vitreous, $80 \%$ of which is comprised by albumin and immunoglobulin, limits the detection of low abundant proteins, which is a major challenge with 2D-PAGE experiments. This is partly overcome by employing affinity chromatography for proteomics of body fluids, which improves the detection of low abundant protein detection by depleting the high abundant proteins (22). Kim et al. identified 346 proteins using immunoaffinity depletion and liquid chromatography-Matrix assisted laser desorption and ionization (LC-MALDI-MS/MS) (23). Murthy et al. identified 1,205 proteins in the normal human vitreous humor using LC-MS/MS, of which 682 proteins were reported for the first time (11). Skeie et al. performed a proteomic analysis of four vitreous substructures (anterior hyaloid, vitreous cortex, vitreous core and vitreous base) in three non-diseased human eyes, using multidimensional LC-MS/ MS (24). They identified a mean of 2,062 proteins, for each substructure, with 278 proteins being unique to anterior hyaloid, 322 to vitreous cortex, 136 to vitreous core, and 128 to vitreous base. They found many of the vitreous proteins to be originating from the retina, such as rhodopsin and phosphodiesterase, suggesting retina as a potential source of inflammatory mediators. Key pathways and networks were represented, including complement pathway, and damage-associated molecular patterns (DAMPs). Presence of antimicrobial proteins, oxidative stress regulation and energy metabolism proteins distributed throughout the vitreous indicated it to be highly active biologically with dynamic interactions with the surrounding tissues. Further, the various intraocular tissues were protected from infection and oxidative stress by the proteins localized to distinct substructures.

\section{Aqueous Humor}

The human aqueous humor contains about 676 nonredundant proteins, and 328 cytokines, chemokines, and receptors (25). Majority of the proteins have catalytic and enzymatic activity. The proteins are low in concentration $(0.1-0.5 \mathrm{mg} / \mathrm{ml})$, and arise from plasma as filtrates, and ciliary body. Richardson et al. used an advanced proteomic approach to characterize the aqueous humor proteome by depleting abundant (albumin) proteins and employing multidimensional protein identification technology (MudPIT) (26). They identified anti-angiogenic, anti-oxidant, and immunoregulatory proteins in the aqueous, with a possible role in supporting avascular tissues.

\section{Tears}

The advancements in MS tools and separation methods have led to an increase in the number of proteins identified in human tears from about 500 to about 1,466 non-redundant proteins, which are secreted by lacrimal glands and epithelial cells of the ocular surface (27). The proteins in high abundance [albumin, lysozyme (LYZ), lactoferrin, and secretory IgA] mask the detection of lower abundant proteins in proteomic studies. Several factors can cause variations in protein profile of tears, such as closed or open eyes, and method of collection (capillary tubes versus Schirmer strips). However, day-to-day variations or fellow eye variations are minimal in tear protein profiles (28).

\section{Cornea}

The involvement of individual layers often in many corneal diseases emphasizes the need for a separate proteomic analysis of different layers of the cornea. Dyrlund et al. identified 3,250 unique Swiss-Prot proteins in human corneas, by using various methods like SDS-PAGE, in-solution digestion, cation exchange chromatography and LC-MS/MS (29). Of these, 2,737 proteins belonged to the epithelium, 1,679 to stroma, and 880 to the endothelium. Of these, 609 (19\%) proteins were common to all three layers, with C-reactive protein being one of them. The most abundant proteins included immunoglobulins, albumin, alpha1-antitrypsin, haptoglobin, complement component 3 and 9, and serotransferrin. Several of the cornea proteins were common to human tears or human aqueous humor, and many are plasma proteins. The study of human corneal proteome has been limited to Fuch's dystrophy, keratoconus lattice and granular corneal dystrophies. Proteomic investigations of majority of the ocular surface diseases have been performed on tears. In Fusarium keratitis, the tear protein profile revealed several differentially expressed proteins (DEPs) in different stages of the infection (early, intermediate and late) (30). Pooled tear samples (five from each group, and from controls) were subjected to DIGE for protein separation, DeCyder software analysis for quantitation, followed by LC-MS/MS. Functional enrichment analysis of DEPs was done by DAVID software.

\section{Lens}

The human lens have the highest protein content in the body. Majority are structural proteins (90\%), the crystallins, occurring in three distinct families ( $\alpha$-, $\beta$-, and $\gamma$-crystallin). Due to their long life and minimal protein turnover, the crystallins provide ideal targets to study post-translational modifications $(31,32)$.

\section{Ciliary Body}

An in-depth proteomic analysis of human ciliary body using LC/ MS/MS identified 2815 proteins (33). While many were plasma proteins, 896 proteins were unique to the ciliary body. Pathway analysis showed expression of glycolysis and gluconeogenesis, EIF2 signaling and ubiquitin pathway. 


\section{Retina}

About 5042 non-redundant proteins have been identified in the vascular endothelial cells of human retina and/or choroid (34). Of 3,454 proteins that were quantifiable, 498 (14.4\%) were differentially expressed among the two cell populations. Angiogenic proteins were identified in both cells, and immunologic proteins were additionally found in retinal cells.

\section{Retinal Pigment Epithelium}

Lipopolysaccharide (LPS) is known to trigger inflammation in the RPE cells. Song et al. used the latest tandem-mass tags (TMTs) label-based approach with high-resolution MS to compare the proteome and phosphoproteome of the LPS-challenged RPE cell line ARPE-19 (human-derived) versus control cells (unexposed) (35). Among the DEPs, the upregulated proteins include those related to amino acid and lipid metabolism, endoplasmic reticulum stress and cell-matrix adhesion. Proteins associated with mitochondrial respiration and cell cycle checkpoint were downregulated. Pathway analysis revealed MAPKK and Wnt/ $\beta$ catenin signalings. The study demonstrated signals expressed by inflamed RPE, providing insight into various RPErelated disorders.

Table 1 summarizes the proteomics of human ocular tissues.

\section{PROTEOMIC CHARACTERIZATION OF UVEITIS IN ANIMAL MODELS}

\section{Experimental Autoimmune Uveitis or EAU}

Animal models of experimental autoimmune uveitis (EAU), a model of non-infectious T-cell mediated autoimmune uveitis, have been used extensively to conduct vitreous proteome studies to explore the immune mechanisms associated with intraocular inflammation. The damage done by oxidative stress induces pathological changes in the retina much before the damage by the infiltrating inflammatory cells, as evident by the mitochondrial retinal DNA damage in early phases of uveitis (36-38). In a mouse model of early EAU, proteomics approach was used to study the mitochondrial protein alterations, using 2D-DIGE and MALDI-TOF MS, followed by validation with Western Blot analysis and real-time PCR (36). Thirteen proteins were differentially expressed in the early EAU mitochondria, as compared to controls, of which nine were upregulated and four were downregulated. The upregulation of crystallins ( $\alpha \mathrm{A}$ and $\beta \mathrm{B} 2)$ and manganese-SOD (MnSOD) indicated their ability to respond to oxidative stress, also offering a protective effect against it. A high metabolic demand and an increased density of mitochondria in the photoreceptors make them susceptible to oxidative stress, which induces an increased expression of $\alpha \mathrm{A}$ crystallins. $\alpha \mathrm{A}$ crystallin prevents the release of cytochrome $c$ into the cytosol by binding with it, and, hence, prevents damage by inhibiting subsequent photoreceptor apoptosis (39). This study demonstrated for the first time the presence of $\beta \mathrm{B} 2$ crystallins in the photoreceptor mitochondria, where they maintain calcium homeostasis and help stabilize mitochondrial proteins. The early molecular damage in mitochondria was evident by the downregulation of ATP synthase, malate dehydrogenase, calretinin, and guanine nucleotide-binding proteins. The morphology and membrane potential of mitochondria are maintained by ATP synthases. Loss of their activity and reduced cellular ATP levels reflect early pathological changes in EAU. The authors concluded that the alterations in mitochondrial protein expression were induced by mitochondrial oxidative stress in the retina during early phases of uveitis.

Subsequently, the authors demonstrated posttranslational modifications in nine out of the 13 DEPs in the retinal mitochondria during early EAU by using MALDI-TOF (38). These modifications included oxidation (ATP synthase, MnSOD), carbamidomethylation ( $\mathrm{mtHsp} 70$, laminin, syntaxin-binding protein, and AT synthase), and pyro-glu modification ( $\alpha \mathrm{A}$ crystallin). Mitochondrial dysfunction is integral to various pathological diseases, and is caused by altered proteins expression and their posttranslational modification. The latter is associated with events like unfolding, degradation, or aggregation of proteins.

Okuniki et al. used proteomic approach to study the role of retinal autoantigens in spreading the ocular inflammation in human endogenous uveoretinitis (40). After inducing EAU in mice, they detected the presence of autoantibodies against the retinal proteins (antigens) by $2 \mathrm{D}$ electrophoresis and western blotting. They identified six new candidate autoantigens in the murine model as the retinal proteins targeted by the autoantibodies using MS, as $\beta$-actin (bAct), esterase D (EsteD), tubulin $\beta-2$, brain-type creatine kinase (BB-CK), voltagedependent anion-selective channel protein, and aspartate

TABLE 1 | Proteomics of human ocular tissues.

\begin{tabular}{|c|c|c|c|c|}
\hline Ocular tissue & $\begin{array}{l}\text { Average protein } \\
\text { concentration }\end{array}$ & Proteins in high abundance & $\begin{array}{l}\text { Number of proteins } \\
\text { identified }\end{array}$ & Study \\
\hline Vitreous humor & $0.5 \mathrm{mg} / \mathrm{ml}$ & Albumin and immunoglobulin & $\begin{array}{c}1,111 \\
346 \\
1,205 \\
2,062\end{array}$ & $\begin{array}{l}\text { Aretz et al. (12) } \\
\text { Kim et al. (23) } \\
\text { Murthy et al. (11) } \\
\text { Skie et al. (24) }\end{array}$ \\
\hline Aqueous humor & $0.1-0.5 \mathrm{mg} / \mathrm{ml}$ & Albumin & 676 & Chowdhury et al. (25) \\
\hline Tears & & Albumin, lysozyme, lactoferrin and secretory lgA & 1,466 & Zhou et al. (27) \\
\hline Cornea & & $\begin{array}{l}\text { Immunoglobulins, albumin, alpha-1-antitrypsin, } \\
\text { haptoglobin, complement component } 3 \text { and } 9 \text {, and serotransferrin }\end{array}$ & 3,250 & Dyrlund et al. (29) \\
\hline Lens & Highest & Crystallins & - & Kyselova et al. (31) \\
\hline Ciliary body & & Plasma proteins & 2,815 & Goel et al. (33) \\
\hline Retina and/or choroid & & Angiogenic proteins & 5,042 & Smith et al. (34) \\
\hline
\end{tabular}


aminotransferase. They further examined immunoreactivity by ELISA against bAct, EsteD, and BB-CK in human endogenous uveitis [36 Behcet's disease (BD), 16 Vogt-Koyanagi-Harada (VKH) disease and 17 sarcoidosis patients]. Of these, EstD and BB-CK reacted with sera from $\mathrm{BD}$ (25\% and 5.6\%, respectively), VKH (25\% each), and sarcoidosis $(17.6 \%$ and $38.4 \%$, respectively), suggestive of autoantigenicity in both EAU and human endogenous uveitis. Despite being a melanocyte-specific autoimmune disease, they demonstrated the additional role of autoimmunity to retinal antigens in VKH disease. This study was the first to identify the role of EstD in autoimmunity in human uveitis. The authors believed that the generation of EstD and BB$\mathrm{CK}$ as retinal autoantigens was secondary to the retinal destruction induced by uveitis, which possibly extended the ocular inflammation in endogenous autoimmune uveitis.

To test the hypothesis that different proteins are expressed in different forms of uveitis, Pepple et al. compared the inflamed aqueous humor of two uveitis models of acute inflammation in Lewis rats [EAU-2 weeks, and primed mycobacterial uveitis (PMU)-2 days] with the naïve aqueous humor by proteomics approach using 2D gel electrophoresis and MALDI-TOF (41). A larger amount of ocular fluid is available in rat than mouse. Innate immunity plays a significant role in an animal model of PMU, as the killed mycobacteria are introduced into the vitreous cavity. They found the total protein concentration to be increased in the inflamed aqueous of both uveitis models, as compared to the naïve aqueous, including calprotectin (a heterodimer of S100A8 and S100A9) and apolipoprotein E. Apolipoprotein E had higher levels in EAU than PMU in the aqueous. The vitreous also showed increased levels of S100A8 in both uveitis models, higher in PMU than EAU. Beta-B2-crystallin was markedly decreased in EAU as compared to PMU and naïve eyes, both in the aqueous and vitreous. This finding was in contrast to the previous reports of upregulation of mitochondrial crystallins (both $\alpha \mathrm{A}$ and $\beta \mathrm{B} 2$ ) during early EAU (36). The authors attributed this to the temporal association of $\beta B 2$ crystallin changes in EAU. The widespread retinal necrosis during "early" EAU caused an increased expression of $\beta B 2$ crystallin (36), which could not be detected during peak inflammation at "two weeks" owing to retinal cell destruction causing background loss of proteins (41). They found that the overall complexity of the protein constituents was decreased in the inflamed aqueous and increased in the inflamed vitreous (as compared to naïve vitreous) due to breakdown of blood-retinal barrier leading to exudation of serum major proteins into the vitreous.

The patterns of EAU induced by opsin or rhodopsin involve dense cellular infiltration in both retinochoroid and anterior uvea, with rhodopsin being more pathogenic than opsin (42-44). Schalken et al. provided the first evidence of EAU induced by purified rhodopsin in monkeys (44). A dense cellular and humoral response occurred before the onset of EAU, indicating the role of cellular immunity in the pathogenesis of EAU.

\section{Endotoxin-Induced Uveitis}

In an acute ocular inflammation model of an animal (endotoxininduced uveitis or EIU), Bahk et al. used proteomic approach (2D gel electrophoresis and micro LC/LC-MS/MS) to compare the protein infiltration in the vitreous of EIU rats with vitreous of normal rats (45). They found crystallins in intact form in EIU rat vitreous, and truncated $\beta \mathrm{A} 4$ - and $\beta \mathrm{B} 2$-crystallin in normal vitreous. They suggested that the crystallins were the predominant proteins involved in EIU, and the specific modifications in crystallin family proteins (such as truncation of C-terminal of $\beta$-crystallins) caused progression of EIUrelated disease.

\section{Equine Recurrent Uveitis}

Equine recurrent uveitis (ERU), a spontaneous model for human autoimmune uveitis, is a blinding, recurrent, lymphocyte-driven, autoimmune disease occurring in horses (46). Proteomic studies of vitreous and retinal samples from ERU eyes versus healthy controls have revealed a list of DEPs, which are associated with maintenance of blood-retinal barrier and immune system (47). In a study by Hauck et al., which compared the retinal proteome of ERU horses with controls (48), glial fibrillary acidic protein (GFAP) was among the several upregulated proteins, which suggested the involvement of retinal Mueller glial cells (RMG) in uveitis. Screening for RMG-specific markers revealed an upregulation of vimentin and GFAP, and downregulation of glutamine synthetase. The subsequent downregulation of pigment epithelium-derived factor (PDEF) by the activated RMGs and expression of pro-inflammatory interferon-gamma indicated a significant role of RMGs in progression of uveitis by triggering interferon-gamma release. Degroote et al. identified 362 spots in the equine lymphocyte proteome by 2D SDS-PAGE (49). When compared with healthy horses, seven DEPs were detected by 2D-DIGE technique, and identified by MS. One (lactotransferrin) was upregulated in ERU lymphocytes while six (voltage-dependent anion-selective channel protein 2, programmed cell death 6-interacting protein, protein tyrosine phosphatase non-receptor type6, glyceraldehyde-3-phosphate dehydrogenase, ezrin and septin 7) were downregulated. A decreased expression of septin 7 in both CD4+ and CD8+ lymphocytes of ERU, with no expression difference in B cells, suggested that the predominant cell types in ERU were the T cells. Septin 7 inhibits cellular proliferation. Its downregulation in ERU activates $\mathrm{T}$ cells and promotes inflammation.

Two novel retinal autoantigens were detected in ERU cases by proteomic approach, namely cellular retinaldehyde-binding protein (CRALBP) and malate dehydrogenase $(\mathrm{MDH})$, both with $\mathrm{B}$ - and $\mathrm{T}$ - cell autoreactivity (50). While $\mathrm{MDH}$ induces uveitis only in Lewis rats (and not in horses), CRALBP is uveitogenic in both rats and horses.

Table 2 summarizes the proteomic characterization of uveitis in animal models.

\section{HUMAN PROTEOMICS IN UVEITIS}

\section{Vitreous and Autoimmune Posterior Uveitis}

Though vitreous proteomics in humans has evolved extensively over the last two decades in healthy eyes as well as in various vitreoretinal disorders, it is still in its infancy in uveitis. Most of 
TABLE 2 | Proteomic Characterization of uveitis in animal models.

\begin{tabular}{|c|c|c|c|c|}
\hline Type of animal model of uveitis & Tissue studied & Proteins targeted & Inference & Study \\
\hline EAU (mice) & Retina & Retinal autoantigens & $\begin{array}{l}\text { Role of retinal autoantigens in spreading the ocular inflammation in } \\
\text { human endogenous autoimmune uveitis }\end{array}$ & Okuniki et al. (40) \\
\hline EAU (monkey) & & Purified rhodopsin & Role of cellular immunity in the pathogenesis of EAU & Schalken et al. (44) \\
\hline $\begin{array}{l}\text { EIU } \\
\text { (rat) }\end{array}$ & Vitreous & & Crystallins were the predominant proteins involved in EIU & Bahk et al. (45) \\
\hline ERU (horse) & Retina & & $\begin{array}{l}\text { Upregulation of GFAP suggested the involvement of retinal Mueller } \\
\text { glial cells (RMG) in uveitis }\end{array}$ & Hauck et al. (48) \\
\hline
\end{tabular}

EAU, Experimental autoimmune uveitis; PMU, primed mycobacterial uveitis; EIU, endotoxin-induced uveitis; ERU, Equine recurrent uveitis; CRALBP, cellular retinaldehyde-binding protein; $\mathrm{MDH}$, malate dehydrogenase.

the proteomics studies on vitreous samples in uveitis so far have been done in animal models, with only a few studies addressing the proteome of human vitreous in uveitis (51-56).

Several vitreous proteins have been reported to be associated with autoimmune uveitis (51). Kasudhan et al. compared the vitreous fluid proteome of infectious (34 samples) and noninfectious (56 samples) uveitis by using SDS-PAGE (which identified 25 different proteins), 2D electrophoresis (which identified 22 proteins), and matrix-assisted laser desorption and ionization- time of flight mass spectrometry (MALDI-TOF MS) (51). Among the proteins identified by SDS-PAGE, half revealed association with heterotrimeric G-Protein signaling pathway-rod outer segment phototransduction, and the other half were associated with de novo purine biosynthesis. Of the proteins identified by $2 \mathrm{D}$ electrophoresis, $20 \%$ showed association with de novo purine biosynthesis. The proteins that were common in both analyses included carbonic anhydrase1 and serpin $\mathrm{B} 3$, both of which are associated with acute anterior uveitis (AAU) and autoimmunity $(57,58)$. About $40 \%$ of the proteins were associated with glycolytic pathway. Five of the proteins identified in their study had associations with uveitis: alpha-1-acid glycoprotein ( $\alpha 1$-AGP) with acute idiopathic anterior uveitis (59), recoverin with autoimmune uveitis (60), selenium-binding protein 1 with $\mathrm{BD}(61)$, perforin with viral uveitis $(62,63)$, and carbonic anhydrase 1 with AAU $(64)$.

Velez et al. reported the use of proteomics in diagnosing a case of idiopathic uveitis as autoimmune retinopathy by profiling the vitreous fluid biopsy for cytokine expression (52). Seventeen upregulated and 12 downregulated cytokine signals were detected, following which the diagnosis was confirmed by the presence of antibodies against S-arrestin. Administration of targeted therapy led to reversal of visual loss, demonstrating the use of proteomics in personalized medicine. They performed cytokine profiling of vitreous fluid in 15 cases of posterior uveitis and five normal controls. Uveitic vitreous had $60 \mathrm{DEPs}$, of which insulin like growth factor-binding protein 2 (IGFBP-2), platelet-derived growth factor receptor $\beta$ polypeptide (PDGFRb), interleukin 23 (IL-23), IL-17R, IL-1 receptor I (IL-1RI), nerve growth factor (bNGF), bone morphogenic protein 4 (BMP-4), tissue inhibitors of metalloproteinase 1 and 2 (TIMP-1 and TIMP-2), and stem cell factor (SCF) were expressed in vitreous of all uveitis cases.

Characterization of uveitis proteome has translational significance and relevance for personalized proteomics (53). Beyond their diagnostic indications, the use of proteomics of ocular fluids is extending into the clinics for personalized management of patients. Detection of a cytokine signature common to all 15 cases with different forms of uveitis [idiopathic posterior uveitis, viral endophthalmitis, autoimmune retinopathy, HLA-B27 uveitis, multifocal choroiditis, and neovascular inflammatory vitreoretinopathy (NIV)] opened the possibility of targeting different diseases with the same therapy (52). In eyes with NIV, an autoinflammatory, hereditary disease with blinding outcome despite intensive therapy, real time proteomics of liquid biopsies in various stages of the disease enabled detection of dynamic events during intraocular inflammation (54). Vitreous profiling was done to analyze 200 cytokine-signaling proteins by an antibody array by comparing NIV (8 eyes) and non-NIV (4 eyes) control eyes. By cytokine array, 64 DEPs were detected (61 upregulated and 3 downregulated). The TNF- $\alpha$ levels were found normal in all NIV eyes. This explained the failure of these eyes to respond to previous treatment with conventional immunosuppression with an anti-TNF- $\alpha$ agent (infliximab). Detection of elevated levels of vascular endothelial growth factor (VEGF) by proteomic analysis guided the anti-VEGF therapy in NIV eyes with successful outcomes, without the need for vitreous surgery for hemorrhagic complications. Pathway analysis revealed significant representation of the mTOR and class I PI3K signaling pathways. Considering the mTOR effectors present in NIV vitreous, 
the authors redirected the previous ineffective oral methotrexate therapy to intravitreal methotrexate therapy to target NIV-specific T cells. A dramatic reduction in anterior chamber inflammation led to the use of intravitreal methotrexate in routine clinics as an adjunct for perioperative management of NIV patients, also avoiding the corticosteroid-related side effects. Recurrent fibrosis could be prevented by anti-IL- 6 therapy.

Velez et al. utilized vitreous proteomic strategy to personalized medicine, emphasizing protein biomarkers for a therapeutic potential, rather than as diagnostic biomarkers (52). They recommend the vitreous protein profiling for a therapeutic approach, to identify the best therapy by targeting proteins that are present in the vitreous, and avoiding the ones that are not found in the vitreous.

\section{Intermediate Uveitis}

Sepah et al. recently reported proteomics screening of vitreous samples of three patients (four eyes) with intermediate uveitis (IU) to discover biomarkers and therapeutic targets, suggesting the role of both innate and adaptive immunity in IU (56). The LCMS/MS approach identified 233 DEPs (103 proteins upregulated 130 downregulated) among IU and control samples. Fibronectin (FN) was among the proteins with highest expression and glutathione synthetase (GSS) among the least expression in IU samples. Known for organizing collagen and extracellular matrix components, an upregulated FN in IU may be responsible for snowballs formation, a finding typical of IU. The downregulated GSS in IU indicates a reduced regulation of oxidative stress. Ingenuity Pathway Analysis (IPA) revealed complement system, coagulation system, glycolysis and IL-12 (with 22 inflammatory mediators) signaling in macrophages among the most represented pathways. The most significantly activated regulators of these pathways were myeloid differentiation primary response protein (MYD88) and IL-23. As both promote myeloid cell recruitment and differentiation, myeloid cell recruitment was found to be an upstream pathway in IU pathogenesis. The upregulated IL-23 in IU vitreous helped CD4+ T cells to differentiate into IL-17 helper T cells, which promote the release of pro-inflammatory mediators. The IL-23/IL-17 pathway is integral to many systemic autoimmune diseases, and its expression in IU suggests the autoimmune pathologic drive in IU. Also, it offers the potential to be a therapeutic target in IU by the biologics.

\section{Juvenile Idiopathic Arthritis-Associated Uveitis}

Several biomarkers (S100, S100A8, S100A9, and S100A12 proteins) have been associated with various aspects of systemic manifestations of Juvenile Idiopathic Arthritis (JIA), in predicting the phenotype, severity, and progression of the disease, for predicting flare of the disease, higher risk of poor response to methotrexate treatment (two single nucleotide polymorphisms or SNPs in the ATIC gene and one SNP in ITPA gene), predicting response to treatment (S100), and disease relapse (high S100A12 and MRP8/14) with six months of discontinuing treatment (65). Similar challenges occur in uveitis associated with JIA, prompting the need for biomarker discovery from ocular fluids.
In the first study of proteomics of aqueous humor of children with uveitis, Ayuso et al. investigated the aqueous and serum samples (paired) of patients with JIA-associated uveitis (62 patients), using SELDI-TOF MS, and compared with silent chronic anterior uveitis (non-infectious) (7 patients), other uveitis entities (27 patients), and non-inflammatory controls (15 patients; pediatric cataract or glaucoma) (66). They found significant differences in the protein profile of JIA-associated uveitis, when compared to other uveitis entities and non-inflammatory controls, but similar protein compositions in aqueous humor of patients with silent chronic AU and JIA-associated uveitis. They hypothesized that patients with chronic silent AU were a subset of JIA, primarily manifesting with uveitis, but without rheumatological signs. A significantly higher concentration of transthyretin (TTT) in aqueous humor of patients with silent chronic AU and JIAassociated uveitis as compared to other groups led to its identification as a potential intraocular biomarker of JIAassociated uveitis. In human eye, TTR is produced by the RPE, and plays a role in the transport of thyroid hormones and retinolbinding protein (RBP). Further, TTT was reported as one of the proteins upregulated in synovial fluid of rheumatoid arthritis patients (67). Subsequently, Clement et al. used biochemical and proteomic approaches to demonstrate an autoimmune response to TTT in JIA, suggesting its role in the pathogenesis of JIA as an autoantigen (68).

This was followed by a pilot study of tears to evaluate for biomarkers in children with chronic non-infectious AU, using LCMS/MS (69). Tears were collected from seven patients using Schirmer strips: three with JIA-associated uveitis (JIA-U), and four with idiopathic chronic anterior uveitis (I-CAU). Various cytokines (S100A8, S100A9, TTR, sCD14, LAP3, and SAA1) that are present in aqueous humor of JIA-U, were detected in tears of all JIA-U and I-CAU children, with a higher expression of S100A8, sCD14, and SAA1 in JIA-U tears, and a lower expression of S100A9, TTR, LAP3, and MIF in JIA-U tears, as compared to I-CAU tears. The authors demonstrated a similar cytokine profile of tears and aqueous in children with CAU. The differential expression of several proteins in tears of JIA-U and I-CAU reflected intrinsic differences between the two phenotypically similar entities.

To study the autoimmune target structures in JIA-associated uveitis, Busch et al. employed 2D-PAGE, western blotting and MS to analyze the binding patterns of serum autoantibodies to proteomes isolated from porcine ocular tissues (iris, ciliary body, and retina) (70). A broad range of serum antibodies were found in JIA-U, mostly directed against iris antigens (vimentin, tubulin beta chain, ATP synthase subunit beta, actin, and L-lactate dehydrogenase $\mathrm{B}$ chain. With iris being the major site of inflammation in JIA-U, these findings support the role of autoantibodies in immunopathology of JIA-associated uveitis.

\section{Behcet's Disease}

$\mathrm{BD}$ is a chronic, autoinflammatory disease with multisystem involvement. Uveitis is chronic, recurrent, bilateral, and sightthreatening. The lack of clinical or diagnostic biomarkers makes it imperative to explore prognostic biomarkers to predict the disease progression and response to therapy. $\mathrm{Hu}$ et al. identified 
CTDP1 (RNA polymerase II subunit A C-terminal domain phosphatase) as a novel BD autoantigen using HuProt arrays (71).

In $\mathrm{BD}$ patients with active uveitis, serum samples were subjected to proteomic analysis using magnetic beads, MALDITOF-MS, and bioinformatics tool, and compared with non-BD patients (VKH disease and healthy controls) (72). Thirty-nine DEPs were detected, which indicated the involvement of unidentified proteins, and a broad change in complemented immunoglobulins and autoantibodies in progression of BD. This differential serum proteomic profile of BD (with 39 differential $\mathrm{m} / \mathrm{z}$ peaks) was used to construct a classification algorithm, based on which $\mathrm{BD}$ could be successfully distinguished from $\mathrm{VKH}$ and controls using a panel of six $\mathrm{m} / \mathrm{z}$ peaks. This diagnostic model had a sensitivity of $84.62 \%$ and specificity of $90.48 \%$ in diagnosing BD, and a sensitivity of $90 \%$ and specificity of $100 \%$ in diagnosing VKH disease. The discovered biomarkers also aided in determining the stages of BD.

The most recent breakthrough in proteomics of uveitis was reported in tears of $\mathrm{BD}$ patients with unilateral relapse of uveitis (73). Fifteen patients with unilateral relapse of $\mathrm{BD}$-associated uveitis (BDU) were enrolled. Tears were collected from both eyes (the diseased eye with relapse of uveitis and fellow quiescent eye), and subjected to data-independent acquisition (DIA) proteomics for protein profiling. Of 1,797 proteins detected, 51 were differentially expressed in uveitic and quiescent eyes, some of which were related to inflammation or immunity. In uveitisrelapsed eyes, $\alpha 1-A G P$, an immunomodulatory protein, was increased. Annexin A1 (ANXA1), an anti-inflammatory protein, and LYZ were decreased. The role of ANXA1 in innate and adaptive immunity is multifunctional and involves resolution of inflammation. This study strengthened the role of tear proteomics as potential sources of biomarkers for uveitis and established $\alpha 1$-AGP and ANXA1 as potential biomarkers for monitoring of uveitis in $\mathrm{BD}$.

\section{ANKYLOSING SPONDYLITIS}

Sera from ankylosing spondylitis (AS) or other autoimmune disease patients were subjected to human protein microarray analysis (74). A significantly higher level of anti-prefoldin subunit 5 (PFDN5) antibodies were found in sera of AS patients with uveitis, as compared to AS without uveitis. To further assess the pathogenic role of this biomarker identified in AS with uveitis, the authors conducted an animal study involving curdlan-induced SKG mice model, which developed uveitis and peripheral arthritis, compared to PBS-treated SKG mice. Half of them developed anterior uveitis at week 8, while all developed uveitis (histologically confirmed) at week 16. The ocular lesions demonstrated apoptosis and an increased PFDN5 expression, and the sera showed increasing levels of anti-PFDN5 antibodies over time. PFDN5 also revealed a protective role against apoptosis of retinal cells, preventing cell death in uveitis.

\section{Acute Anterior Uveitis}

Tear samples from five patients with unilateral AAU were subjected to proteomics using LC-MS/MS to detect any ipsilateral changes in tear proteome, with fellow unaffected eyes serving as controls (75). Of 242 identified proteins in both groups of eyes, 32 tear proteins were detected in all five patients. Nine proteins were upregulated and eight downregulated in uveitis eyes as compared to normal eye. The diseased eyes showed a significant increase in Apolipoprotein B mRNA editing enzyme catalytic subunit 3A (APOBEC3A), and a significant decrease in transglutaminase 2 (TGM2). The top canonical pathways identified by IPA to be affected in tears included the liver X receptor/retinoid X receptor (LXR/RXR) and the farsenoid $X$ receptor/retinoid $X$ receptor (FXR/RXR). IL-6 and tumor necrosis factor (TNF) were identified as possible upstream regulators. The study showed that the tears expressed ipsilateral proteomic changes in unilateral AAU, which were detectable by MS.

\section{Human Uveitis Associated With Oral Moxifloxacin Therapy}

Oral moxifloxacin therapy is associated with bilateral panuveitis with anterior chamber pigment dispersion $(76,77)$. In a case report by Hinkle et al., aqueous humor samples were obtained from both eyes of a patient with bilateral uveitis-like syndrome with pigment dispersion during acute phase, and one eye of a normal subject who consented for aqueous harvest prior to cataract surgery. Moxifloxacin concentration was tested by multiple reaction monitoring (MRM) MS. Proteomic analysis was done using shotgun approach via LC-MS/MS (78). Eighteen days after completion of oral intake, aqueous humor demonstrated higher than expected levels of moxifloxacin. The aqueous of affected eyes revealed 33 proteins, and that of unaffected (control) eye showed 32 proteins. Ten proteins were significantly downregulated in uveitis eyes.

\section{VKH Disease}

The role of CD4+ T cells in autoimmune diseases including VKH disease is well known. Mao et al. used label-free quantitative proteomic strategy to detect DEPs between VKH patients and healthy controls (79). They tested blood samples of five active $\mathrm{VKH}$ patients and five healthy controls. Three hundred fifty-four peptides were differentially expressed between the two groups, of which 102 could be sequenced, corresponding to 64 proteins. A difference of at least 1.5 -fold was seen in 30 proteins between $\mathrm{VKH}$ patients and normal controls. Among these, the expression of two proteins, CD18 and AKNA, was more than three-fold lower in active VKH patients compared to controls. These proteins were further validated in other five active $\mathrm{VKH}$ patients and six normal subjects by Western blot assay. As CD18 induces apoptosis of human $\mathrm{T}$ cells, its reduction in active $\mathrm{VKH}$ disease suggests a reduced inhibition of CD4+ T cell apoptosis. The observations of this study open the research on the role of these proteins in influencing apoptosis of CD4+ T cells and cytokine expression. 


\section{Human Autoimmune Uveitis and CRALBP}

The role of CRALBP as an autoantigen is well known in ERU. Its role in human autoimmune uveitis was tested by Deeg et al., using 2D western blotting, followed by SDS-PAGE and MS for identification of immunoreactive proteins (80). Sera from 56 uveitis patients and 23 healthy subjects (controls) were used. The normal equine retinal proteome was used as a source of antigen in 2D Western blots, where human uveitis sera were made to react with CRALBP. CRALBP expression pattern in normal eyes was evaluated by using six human donor eyes (normal with no signs of uveitis), displaying a strong CRALBP expression in Mueller glial cells. Anti-CRALBP autoantibodies were found in 30 (54\%) out of 56 uveitis sera, and in four (17.4\%) out of 23 control sera $(\mathrm{p}<0.01)$, suggesting the role of CRALBP as a potential autoantigen in human autoimmune uveitis.

Table 3 summarizes the proteomics of various types of human uveitis.

\section{OCULAR SAMPLING TECHNIQUES FOR PROTEOMICS}

Procurement of surgical specimens is critical for proteomic analysis, along with their handling and storage. The invasive nature of vitreous and aqueous sampling restricted the research in human subjects till few decades ago. Skeie et al. reported comparable yields of vitreous needle biopsies and vitrectomy (cutter) biopsies (81). They compared 23-gauge needle method with 23-gauge vitreous cutter method performed sequentially for vitreous biopsy in 10 eyes. The UV absorbance spectroscopy revealed equivalent protein concentrations in paired samples. The SDS-PAGE revealed similar molecular weight protein profiles, and LC-MS/MS identified equivalent peptides in paired samples, with only a few proteins and peptides differing in concentration. The aspiration method has the advantages of being safe and reproducible, yields undiluted vitreous and can be

TABLE 3 | Proteomic studies in various types of human uveitis.

\begin{tabular}{|c|c|c|c|c|}
\hline Type of uveitis & Tissue studied & Differentially expressed proteins & Enriched pathway & Study \\
\hline $\begin{array}{l}\text { Autoimmune posterior } \\
\text { uveitis }\end{array}$ & Vitreous & $\begin{array}{l}\text { alpha-1-acid glycoprotein, recoverin, selenium-binding protein 1, } \\
\text { perforin, carbonic anhydrase } 1\end{array}$ & glycolytic pathway & $\begin{array}{l}\text { Kasudhan et al. } \\
(51)\end{array}$ \\
\hline Posterior uveitis & Vitreous & $\begin{array}{l}\text { insulin like growth factor-binding protein } 2 \text { (IGFBP-2), platelet- } \\
\text { derived growth factor receptor } \beta \text { polypeptide (PDGFRb), interleukin }\end{array}$ & & Velez et al. (52) \\
\hline
\end{tabular}
derived growth factor receptor $\beta$ polypeptide (PDGFRb), interleukin 23 (IL-23), IL-17R, IL-1 receptor I (IL-1RI), nerve growth factor (bNGF), bone morphogenic protein 4 (BMP-4), tissue inhibitors of metalloproteinase 1 and 2 (TIMP-1 and TIMP-2), and stem cell factor (SCF)

Neovascular inflammatory Vitreous vitreoretinopathy (NIV) Intermediate uveitis

Vitreous
Human uveitis associated with oral Moxifloxacin

therapy Vogt-Koyanagi-Harada disease

Human autoimmune Serum uveitis
Elevated vascular endothelial growth factor (VEGF) levels; normal TNF- $\alpha$ levels

233 DEPs (103 upregulated and 130 downregulated); Fibronectin, glutathione synthetase

Transthyretin (TाT)

Cytokines (S100A8, S100A9, TTR, sCD14, LAP3 and SAA1)

39 DEPs;

CTDP1 (RNA polymerase II subunit A C-terminal domain phosphatase) as a novel BD autoantigen in Behcet's disease Alpha-1-acid glycoprotein 1 ( $\alpha 1$-AGP), Annexin A1 (ANXA1) anti-prefoldin subunit 5 (PFDN5) antibodies 17 DEPs;

Apolipoprotein B mRNA editing enzyme catalytic subunit 3A (APOBEC3A) (upregulated), transglutaminase 2 (TGM2) (downregulated)

higher than expected levels of moxifloxacin

30 DEPs; CD18 and AKNA

Anti-CRALBP autoantibodies
mTOR and class I PI3K signaling pathways complement system, coagulation system, glycolysis and IL-12 (with 22 inflammatory mediators) signaling in macrophages Biomarker in JIA

Ayuso et al. (66)

Angeles-Han et al. (69) Hu et al. (71)

Innate and adaptive immunity liver $\mathrm{X}$ receptor/retinoid $\mathrm{X}$ receptor ( $L X R / R X R)$ and the farsenoid $X$ receptor/retinoid $X$ receptor (FXR/RXR)

Apoptosis of human T cells

Mao et al. (79) role of CRALBP as a potential Deeg et al. (80) autoantigen in human autoimmune uveitis

Liang et al. (73) Kwon et al. (74) Eidet et al. (75)

Hinkle et al. (78)

DEPs, Differentially expressed proteins 
performed in an office-based setting under local anesthesia (82, 83). However, the limited sample volume is a major limitation for performing large-scale proteomic studies. On the other hand, vitreous biopsy cutter method yields adequate volume of sample and reduces the chances of hypotony. It is, however, more invasive and requires vitreous surgery in the form of pars plana vitrectomy (PPV) in an operating room. Vitreous needle biopsy by a fine needle aspiration was the least complex and most followed method, until the advent of microincision vitreous surgery (MIVS). The liquid vitreous specimens transported to the laboratory are centrifuged to separate the cellular debris (pellet) and supernatant. While pellet is subjected to DNA-based molecular tests, the supernatant is cryopreserved at $-80^{\circ} \mathrm{C}$ for future research studies, including proteomics. Though the safety and efficacy of PPV is now well established in uveitis in the era of MIVS $(84,85)$, their contribution toward proteomic quality of vitreous samples is yet to be addressed.

Sampling of aqueous humor in uveitis is indicated in anterior uveitis, or in cases where PPV is not indicated. The human aqueous humor of healthy controls is typically sampled during cataract surgery. The study eye with uveitis usually undergoes an aqueous biopsy or an anterior chamber tap in an operating room $(25,86)$. A 25 - or 26 - or 30 - gauge needle is inserted in to the anterior chamber at the limbus through the peripheral cornea through a paracentesis tract, and about $100 \mu \mathrm{l}$ of the aqueous fluid can be aspirated until the shallowing of anterior chamber starts to occur.

Tear protein profiles have been characterized in several inflammatory and non-inflammatory ocular disorders, using MALDI-TOF, surface-enhanced laser desorption/ionization time-of-flight mass spectrometry (SELDI-TOF-MS) and LCMS. A relatively high concentration of proteins in the tears along with the non-invasive nature of tear sample collection make tears a potential source for biomarker discovery in proteomic studies (87-89).

\section{CLINICAL APPLICATIONS AND FUTURE DIRECTIONS IN PROTEOMICS OF UVEITIS}

The discovery of biomarkers in uveitis is showing a promising role in clinical practice. The diagnosis of autoimmune retinopathy, a rare uveitis entity with a diagnostic and therapeutic challenge, was made possible in a case of idiopathic uveitis by cytokine profiling of the vitreous, which demonstrated the presence of antibodies against S-arrestin (52). Normal levels of TNF- $\alpha$ in the vitreous of NIV eyes explained the reason for failure of NIV eyes to respond to treatment with anti-TNF- $\alpha$ agent (54). In the absence of clinical or diagnostic biomarkers, the identification of protein biomarkers in serum and tears of BD patients provided valuable clues in differentiating this disease from another autoimmune disease (VKH disease), helped in staging the disease based on serum biomarkers, and guided in monitoring therapy for uveitis based on tear biomarkers $(72,73)$.
Different forms of autoimmune uveitis can be targeted with the same therapy with the identification of a protein/cytokine signature common to all of them (52).

A differential protein expression in the intraocular fluids of patients with two phenotypically similar entities has significant clinical implications. Transthyretin (TTT), a potential intraocular biomarker, found in the aqueous humor of silent chronic AU and JIA-associated uveitis patients, provided a common underlying etiology between the two conditions (66).

Tubercular uveitis, a common uveitis entity in endemic countries, poses several clinical and diagnostic challenges, leading to a delay in initiating targeted therapy. The use of proteomics to provide biomarkers in differentiating this disease from other forms of infective and non-infective uveitis is strongly indicated and is a subject of concern.

With the major goal of identifying biomarkers of a disease, proteomics provides information about the changes in protein expression levels between the diseased and normal states, posttranslational modifications, and response to treatment. A new insight into the pathogenesis and course of the disease is obtained by identification of DEPs, and their pathway analysis. The protein constituents identified in a diseased eye can serve as potential targets for therapeutic intervention by the drugs that are available and accessible. Also, proteomics provides information about the drugs to be avoided. Besides their impact on diagnosis and treatment of a disease, proteomics may also have implications in gene therapy trials.

\section{SUMMARY}

Considering the various EAU and EIU studies, crystallins may serve as suitable targets for adjunctive therapy in acute phases of uveitis, as also seen by their role in apoptosis and neuroprotection. The ocular proteome in acute uveitis is likely to differ considerably from that of chronic uveitis, addressing the need for exploring and targeting different proteins in different stages of uveitis. With a potential to redirect the diagnosis of idiopathic uveitis, ocular proteomics provide a new insight into the pathophysiology and therapeutics of various ocular inflammatory diseases. Tears, aqueous, and vitreous humor represent potential repositories for proteomic biomarkers discovery in uveitis. Determining the molecular pathways enriched in uveitis helps in considering the drugs that are available and could readily target the pathways, rather than finding new drugs.

\section{AUTHOR CONTRIBUTIONS}

RB has contributed in literature search, and manuscript writing. AG has contributed in manuscript writing and refining. All authors contributed to the article and approved the submitted version. 


\section{REFERENCES}

1. Zhang X, Leung SM, Morris CR, Shigenaga MK. Evaluation of a novel, integrated approach using functionalized magnetic beads, bench-top MALDITOF-MS with prestructured sample supports, and pattern recognition software for profiling potential biomarkers in human plasma. J Biomol Tech (2004) 15:167-75.

2. Röcken C, Ebert MP, Roessner A. Proteomics in pathology, research and practice. Pathol Res Pract (2004) 200:69-82. doi: 10.1016/ j.prp.2004.03.002

3. Baumann S, Ceglarek U, Fiedler GM, Lembcke J, Leichtle A, Thiery J. Standardized approach to proteome profiling of human serum based on magnetic bead separation and matrix assisted laser desorption/ionization time-of-flight mass spectrometry. Clin Chem (2005) 51:973-80. doi: 10.1373/ clinchem.2004.047308

4. Steely HTJr, Clark AF. The use of proteomics in ophthalmic research. Pharmacogenomics (2000) 1:267-80. doi: 10.1517/14622416.1.3.267

5. Lam TC, Chun RK, Li KK, To CH. Application of proteomic technology in eye research: a mini review. Clin Exp Optom (2008) 91:23-3. doi: 10.1111/ j.1444-0938.2007.00194.x

6. Agrawal R, Iyer J, Connolly J, Iwata D, Teoh S. Cytokines and Biologics in non-infectious autoimmune uveitis: Bench to Bedside. Indian J Ophthalmol (2014) 62:74-81. doi: 10.4103/0301-4738.126187

7. Aebersold R, Bader GD, Edwards AM, van Eyk JE, Kussmann M, Qin J, et al. The biology/disease-driven human proteome project (B/D-HPP): enabling protein research for the life sciences community. J Proteome Res (2013) 12:237. doi: $10.1021 / \mathrm{pr} 301151 \mathrm{~m}$

8. Semba RD, Enghild JJ, Venkatraman V, Dyrlund TF, van Eyk JE. The Human Eye Proteome Project: Perspectives on an emerging proteome. Proteomics (2013) 13:2500-11. doi: 10.1002/pmic.201300075

9. Gritz DC, Wong IG. Incidence and prevalence of uveitis in Northern California; The Northern California Epidemiology of Uveitis Study. Ophthalmology (2004) 111:491-500. doi: 10.1016/j.ophtha.2003.06.014

10. Rathinam SR, Namperumalsamy P. Global variation and pattern changes in epidemiology of uveitis. Indian J Ophthalmol (2007) 55:173-83. doi: 10.4103/ 0301-4738.31936

11. Murthy KR, Goel R, Subbannayya Y, Jacob HKC, Murthy PR, Manda SS, et al. Proteomic analysis of human vitreous humor. Clin Proteomics (2014) 11:29. doi: 10.1186/1559-0275-11-29

12. Aretz S, Krohne TU, Kammerer K, Warnken U, Hotz-Wagenblatt A, Bergmann $\mathrm{M}$, et al. In-depth mass spectrometric mapping of the human vitreous proteome. Proteome Sci (2013) 11:22. doi: 10.1186/1477-5956-11-22

13. Holekamp NM. The vitreous gel: more than meets the eye. Am J Ophthalmol (2010) 149:32-6. doi: 10.1016/j.ajo.2009.07.036

14. Wu CW, Sauter JL, Johnson PK, Chen CD, Olsen TW. Identification and localization of major soluble vitreous proteins in human ocular tissue. Am J Ophthalmol (2004) 137:655-61. doi: 10.1016/j.ajo.2003.11.009

15. Skeie JM, Mahajan VB. Dissection of human vitreous body elements for proteomic analysis. J Visualized Exp (2011) 47:2455. doi: $10.3791 / 2455$

16. Shitama T, Hayashi H, Noge S, Uchio E, Oshima K, Haniu H, et al. Proteome profiling of vitreoretinal diseases by cluster analysis. Proteomics-Clinical Appl (2008) 2:1265-80. doi: 10.1002/prca.200800017

17. Funatsu H, Yamashita T, Yamashita H. Vitreous fluid biomarkers. Adv Clin Chem (2006) 42:111-6. doi: 10.1016/S0065-2423(06)42004-7

18. Zhang Y, Fonslow BR, Shan B, Baek MC, Yates III JR. Protein analysis by Shotgun/Bottom-up proteomics. Chem Rev (2013) 113:2343-94. doi: 10.1021/ cr3003533

19. Marcotte EM. How do shotgun proteomics algorithms identify proteins? Nat Biotechnol (2007) 25:755-7. doi: 10.1038/nbt0707-755

20. Mandal N, Heegaard S, Prause JU, Honore B, Vorum H. Ocular proteomics with emphasis on two-dimensional gel electrophoresos and mass spectrometry. Biol Procedures Online (2010) 12:1. doi: 10.1007/s12575-0099019-7

21. Gao BB, Chen X, Timothy N, Aiello LP, Feener EP. Characterization of the vitreous proteome in diabetes without diabetic retinopathy and diabetes with proliferative diabetic retinopathy. J Proteome Res (2008) 7:2516-25. doi: $10.1021 / \mathrm{pr} 800112 \mathrm{~g}$
22. Angi M, Kalirai H, Coupland SE, Damato BE, Semeraro F, Romano MR. Proteomic analyses of the vitreous humor. Mediators Inflamm (2012) 2012:7. doi: 10.1155/2012/148039 Article ID 148039.

23. Kim T, Kim SJ, Kim K, Kang UB, Lee C, Park KS, et al. Profiling of vitreous proteomes from proliferative diabetic retinopathy and non-diabetic patients. Proteomics (2007) 7:4203-15. doi: 10.1002/pmic.200700745

24. Skeie JM, Roybal CN, Mahajan VB. Proteomic insight into the molecular function of the vitreous. PLoS One (2015) 10(5):e0127567. doi: 10.1371/ journal.pone. 0127567

25. Chowdhury UR, Madden BJ, Charlesworth MC, Fautsch MP. Proteome analysis of human aqueous humor. Invest Ophthalmol Vis Sci (2010) 51:4921-31. doi: 10.1167/iovs.10-5531

26. Richardson MR, Price MO, Price FW, Pardo JC, Grandin JC, You J, et al. Proteomic analysis of human aqueous humor using multidimensional protein identification technology. Mol Vision (2009) 15:2740-50.

27. Zhou L, Zhao SZ, Koh SW, Chen L, Vaz C, Tanavde V, et al. In-depth analysis of the human tear proteome. J Proteomics (2012) 75:3877-85. doi: 10.1016/ j.jprot.2012.04.053

28. Gonzalez N, Iloro I, Duran JA, Elortza F, Suarez T. Evaluation of inter-day and inter-individual variability of tear peptide/protein profiles by MALDI-TOF MS analyses. Mol Vis (2012) 18:1572-82.

29. Dyrlund TF, Poulsen ET, Scavenius C, Nikolajsen CL, Thogersen IB, Vorum $\mathrm{H}$, et al. Human cornea proteome: Identification and quantitation of the proteins of the three main layers including epithelium, stroma and endothelium. J Proteome Res (2012) 11:4231-9. doi: 10.1021/pr300358k

30. Ananthi S, Prana NV, Lalitha P, Valarnila M, Dharmalingam K. Pathogen induced changes in the protein profile of human tears from Fusarium keratitis patients. PLoS One (2013) 8(1):e53018. doi: 10.1371/ journal.pone. 0053018

31. Kyselova Z. Mass spectrometry-based proteomics approaches applied in cataract research. Mass Spectrom Rev (2011) 30:1173-84. doi: 10.1002/ mas. 20317

32. MacCoss MJ, McDonald WH, Saraf A, Sadygov R, Clark JM, Tasto JJ, et al. Shotgun identification of protein modifications from protein complexes and lens tissues. Proc Natl Acad Sci U S A (2002) 99:7900-5. doi: 10.1073/ pnas. 122231399

33. Goel R, Murthy KR, Srikanth SM, Pinto SM, Bhattacharjee M, Kelkar DS, et al. Characterizing the normal proteome of human ciliary body. Clin Proteomics (2013) 10:9. doi: 10.1186/1559-0275-10-9

34. Smith JR, David LL, Appukuttan B, Wilmarth PA. Angiogenic and immunologic proteins identified by deep proteomic profiling of human retinal and choroidal vascular endothelial cells: potential targets for new biologic drugs. Am J Ophthalmol (2018) 193:197-229. doi: 10.1016/ j.ajo.2018.03.020

35. Song J, Han D, Lee H, Kim DJ, Cho JY, Park JH, et al. A comprehensive proteomic and phosphoproteomic analysis of retinal pigment epithelium reveals multiple pathway alterations in response to the inflammatory stimuli. Int J Mol Sci (2020) 21:3037. doi: 10.3390/ijms21093037

36. Saraswathy S, Rao NA. Mitochondrial proteomics in experimental autoimmune uveitis oxidative stress. Invest Ophthalmol Vis Sci (2009) 50:5559-66. doi: 10.1167/iovs.08-2842

37. Khurana RN, Parikh JG, Saraswathy S, Wu GS, Rao NA. Mitochondrial oxidative DNA damage in experimental autoimmune uveitis. Invest Ophthalmol Vis Sci (2008) 49:3299-304. doi: 10.1167/iovs.07-1607

38. Saraswathy S, Rao NA. Posttranslational modification of differentially expressed mitochondrial proteins in the retina during early experimental autoimmune uveitis. Mol Vision (2011) 17:1814-21.

39. Rao NA, Saraswathy S, Wu GS, Katselis GS, Wawrousek EF, Bhat S. Elevated retina-specific expression of the small heat shock protein, $\alpha \mathrm{A}$-crystallin, is associated with photoreceptor protection in experimental uveitis. Invest Ophthalmol Vis Sci (2008) 49:1161-70. doi: 10.1167/iovs.07-1259

40. Okunuki Y, Usui Y, Kezuka T, Hattori T, Masuko K, Nakamura H, et al. Proteomic surveillance of retinal autoantigens in endogenous uveitis: implication of esterase D and brain-type creatine kinase as novel autoantigens. Mol Vision (2008) 14:1094-104.

41. Pepple KL, Rotkis L, Wilson L, Sandt A, van Gelder RN. Comparative proteomic analysis of two uveitis models in Lewis rats. Invest Ophthalmol Vis Sci (2015) 56:8449-56. doi: 10.1167/iovs.15-17524 
42. Broekhuyse RM, Kuhlmann ED, van Vugt AH, Winkens HJ. Immunological and immunopathological aspects of opsin-induced uveoretinitis. Graefes Arch Clin Exp Ophthalmol (1987) 255:45-9. doi: 10.1007/BF02155803 225-45-9.

43. Schalken JJ, Van Vugt AH, Winkens HJ, Bovee-Geurts PH, De Grip WJ, Broekhuyse RM. Experimental autoimmune uveoretinitis in rats induced by rod visual pigment: rhodopsin is more pathogenic than opsin. Graefes Arch Clin Exp Ophthalmol (1988) 226:255-61. doi: 10.1007/BF02181192

44. Schalken JJ, Winkens HJ, Van Vugt AH, De Grip WJ, Broekhuyse RM. Rhodopsin-induced experimental autoimmune uveoretinitis in monkeys. $\mathrm{Br} \mathrm{J}$ Ophthalmol (1989) 73:168-72. doi: 10.1136/bjo.73.3.168

45. Bahk SC, Lee SH, Jang JU, Choi CU, Lee BS, Chae SC, et al. Identification of crystallin family proteins in vitreous body in rat endotoxin-induced uveitis: Involvement of crystallin truncation in uveitis pathogenesis. Proteomics (2006) 6:3436-44. doi: 10.1002/pmic.200500779

46. Deeg CA. Ocular immunology in equine recurrent uveitis. Vet Ophthalmol (2008) 1(Suppl 1):61-5. doi: 10.1111/j.1463-5224.2008.00625.x

47. Deeg CA, Hauck SM, Amann B, Pompetzki D, Altmann F, Raith A, et al. Equine recurrent uveitis: a spontaneous horse model of uveitis. Ophthalmic Res (2008) 40(3-4):151-3. doi: 10.1159/000119867

48. Hauck SM, Schoeffmann S, Amann B, Stangassinger M, Gergards H, Ueffing M, et al. Retinal Mueller glial cells trigger the hallmark inflammatory process in autoimmune uveitis. J Proteome Res (2007) 6:2121-31. doi: 10.1021/pr060668y

49. Degroote RL, Hauck SM, Amann B, Hirmer S, Ueffing M, Deeg CA. Unraveling the equine lymphocyte proteome: Differential Septin 7 expression associates with immune cells in equine recurrent uveitis. PLoS One (2014) 9(3):e91684. doi: 10.1371/journal.pone.0091684

50. Deeg CA. A proteomic approach for studying the pathogenesis of spontaneous equine recurrent uveitis (ERU). Vet Immunol Immunopathol (2009) 128(1-3):132-6. doi: 10.1016/j.vetimm.2008.10.302

51. Kasudhan KS, Sarkar S, Gupta V, Gupta A, Chakraborti A. Identification of unique proteins in vitreous fluid of patients with noninfectious uveitis. Acta Ophthalmol (2018) 96:e989-e1003. doi: 10.1111/aos.13801

52. Velez G, Roybal CN, Colgan D, Tsang SH, Bassuk AG, Mahajan VB. Precision Medicine: Personalized proteomics for the diagnosis and treatment of idiopathic inflammatory disease. JAMA Ophthalmol (2016) 134:444-8. doi: 10.1001/jamaophthalmol.2015.5934

53. Velez G, Tang PH, Cabral T, Cho GY, Machlab DA, Tsang SH, et al. Personalized proteomics for precision health: Identifying biomarkers of vitreoretinal disease. Trans Vis Sci Tech (2018) 7:12. doi: 10.1167/tvst.7.5.12

54. Velez G, Bassuk AG, Colgan D, Tsang SH, Mahajan VB. Therapeutic drug repositioning using personalized proteomics of liquid biopsies. JCI Insight (2017) 2:e97818. doi: 10.1172/jci.insight.97818

55. Mahajan VB, Skeie JM. Translational vitreous proteomics. Proteomics Clin Appl (2014) 8:204-8. doi: 10.1002/prca.201300062

56. Sepah YJ, Velez G, Tang PH, Yang J, Chemudupati T, Li AS, et al. Proteomic analysis of intermediate uveitis suggests myeloid cell recruitment and implicates IL-23 as a therapeutic target. Am J Ophthalmol Case Rep (2020) 18:100646. doi: 10.1016/j.ajoc.2020.100646

57. Vidalino L, Doria A, Quarta S, Zen M, Gatta A, Pontisso P. SERPINB3, apoptosis and autoimmunity. Autoimmun Rev (2009) 9:108-12. doi: 10.1016/ j.autrev.2009.03.011

58. Adamus G, Karren L. Against carbonic anhydrase II affects retinal cell functions in autoimmune retinopathy. J Autoimmun (2009) 32:133-9. doi: 10.1016/j.jaut.2009.02.001

59. Gupta AK, Sarin GS, Rastogi A, Grover AK, Pandey PK. Serum alpha-1 acid glycoprotein levels in acute idiopathic anterior uveitis. Ann Ophthalmol (1991) 23:406-9.

60. Whitcup SM, Vistica BP, Milam AH, Nussenblatt RB, Gery I. Recoverinassociated retinopathy: a clinically and immunologically distinctive disease. Am J Ophthalmol (1998) 126:230-7. doi: 10.1016/S0002-9394(98)00149-4

61. Okuniki Y, Usui Y, Takeuchi M, Kezuka T, Hattori T, Masuko K, et al. Proteomic surveillance of autoimmunity in Behcet's disease with uveitis: selenium binding protein is a novel autoantigen in Behcet's disease. Exp Eye Res (2007) 84:823-31. doi: 10.1016/j.exer.2007.01.003

62. Dix RD, Podack ER, Cousins SW. Loss of the perforin cytotoxic pathway predisposes mice to experimental cytomegalovirus virus retinitis. $J$ Virol (2003) 77:3402-8. doi: 10.1128/JVI.77.6.3402-3408.2003
63. Young LH, Foster CS, young JD. In vivo expression of perforin by natural killer cells during a viral infection. Studies on uveitis produced by herpes simplex virus type I. Am J Pathol (1990) 136:1021-30.

64. Turk A, Aykut M, Akyol N, Kola M, Mentese A, Sumer A, et al. Serum anticarbonic anhydrase antibodies and oxidant-antioxidant balance in patients with acute anterior uveitis. Ocul Immunol Inflammation (2014) 22:127-32. doi: 10.3109/09273948.2013.830753

65. Duurland CL, Wedderburn LR. Current developments in the use of biomarkers for juvenile idiopathic arthritis. Curr Rheumatol Rep (2014) 16:406. doi: 10.1007/s11926-013-0406-3

66. Ayuso VK, de Boer JH, Byers HL, Coulton GR, Dekkers J, de Visser L, et al. Intraocular biomarker identification in uveitis associated with juvenile idiopathic arthritis. Invest Ophthalmol Vis Sci (2013) 54:3709-20. doi: $10.1167 /$ iovs.12-10865

67. Ni M, Wei W, Feng Q, Sun XG, Wang YC, Gu YJ, et al. Transthyretin as a potential serological marker for the diagnosis of patients with early rheumatoid arthritis. Clin Exp Rheumatol (2013) 31:394-9.

68. Clement CC, Moncrieffe H, Lele A, Janow G, Becerra A, Bauli F, et al. Autoimmune response to transthyretin in juvenile idiopathic arthritis. JCI Insight (2016) 1(2):e85633. doi: 10.1172/jci.insight.85633

69. Angeles-Han ST, Yeh S, Patel P, Duong D, Jenkins K, Rouster-Stevens KA, et al. Discovery of tear biomarkers in children with chronic non-infectious anterior uveitis: a pilot study. J Ophthal Inflamm Infect (2018) 8:17. doi: 10.1186/s12348-018-0156-5

70. Busch M, Wefelmeyer KL, Walscheid K, Rothaus K, Bauer D, Deeg CA, et al. Identification of ocular autoantigens associated with juvenile idiopathic arthritis-associated uveitis. Front Immunol (2019) 10:1793. doi: 10.3389/ fimmu. 2019.01793

71. Hu CJ, Pan JB, Song G, Wen XT, Wu ZY, Chen S, et al. Identification of novel biomarkers for Behcet disease diagnosis using human proteome microarray approach. Mol Cell Proteomics (2017) 16:147-56. doi: 10.1074/mcp. M116.061002

72. Wang X, Zhang MF, Xie J, Li ZL, Wang P. Behcet's disease with active uveitis: detection of serum protein biomarkers using MALDI-TOF-MS. Anat Rec (2012) 295:1168-73. doi: 10.1002/ar.22502

73. Liang A, Qin W, Zhang M, Gao F, Zhao C, Gao Y. Profiling tear proetomes of patients with unilateral relapsed Behcet's disease-associated uveitis using dataindependent acquisition proteomics. PeerJ (2020) 8:e9250. doi: 10.7717/ peerj. 9250

74. Kwon OC, Lee EJ, Lee JY, Youn J, Kim TH, Hong S, et al. Prefoldin 5 and antiprefoldin 5 antibodies as biomarkers for uveitis in Ankylosing Spondylitis. Front Immunol (2019) 10:384. doi: 10.3389/fimmun.2019.00384

75. Eidet JR, Jorstad OK, Fostad IG, Olstad OK, Sorland RO, Moe MC, et al. Unilateral acute anterior uveitis is associated with ipsilateral changes in the tear fluid proteome that involves the LXR/RXR pathway. $J$ Ophthalmol Inflamm Infect (2020) 10:1-13. doi: 10.1186/s12348-020-00204-4

76. Bringas CR, Iglesias CD. Acute and bilateral uveitis secondary to moxifloxacin. Arch Soc Esp Oftalmol (2004) 79:357-9. doi: 10.4321/S036566912004000700011

77. Wefers BRM, Brouwers K, van langenhove L, De Waard PWT, Missotten TO, Ciriano JPM, et al. Uveitis-like syndrome and iris transillumination after the use of oral moxifloxacin. Eye (Lond) (2009) 23:2260-2. doi: 10.1038/ eye.2009.234

78. Hinkle DM, Kruh-Garcia NA, Kruh JN, Broccardo C, Doctor P, Foster CS. Moxifloxacin concentration and proteomic analysis of aqueous humor in human uveitis associated with oral moxifloxacin therapy. Open Ophthalmol J (2017) 11:107-16. doi: 10.2174/1874364101711010107

79. Mao L, Yang P, Hou S, Li F, Kijlstra A. label-free proteomics reveals decreased expression of $\mathrm{CD} 18$ and AKNA in peripheral CD4+ T cells from patients with Vogt-Koyanagi-Harada Syndrome. PLoS One (2011) 6(1):e14616. doi: 10.1371/journal.pone.0014616

80. Deeg CA, Raith AJ, Amann B, Crabb JW, Thurau SR, Hauck SM, et al. CRALBP is a highly prevalent autoantigen for human autoimmune uveitis. Clin Dev Immunol (2007) 2007:39245. doi: 10.1155/2007/39245

81. Skeie JM, Brown EM, Martinez HD, Russell SR, Birkholz ES, Folk JC, et al. Proteomic analysis of vitreous biopsy techniques. Retina (2012) 32:2141-9. doi: 10.1097/IAE.0b013e3182562017 
82. Pfahler SM, Brandford AN, Glaser BM. A prospective study of in-office diagnostic vitreous sampling in patients with vitreoretinal pathology. Retina (2009) 29:1032-5. doi: 10.1097/IAE.0b013e3181a2cleb

83. Ghodsara DH, Fante R, Gardner TW, Langue M, Niziol LM, Besirli C, et al. Safety and feasibility of quantitative multiplexed cytokine analysis from officebased vitreous aspiration. Invest Ophthalmol Vis Sci (2016) 57:3017-23. doi: 10.1167/iovs.15-18721

84. Bansal R, Gupta A, Gupta V, Mulkutkar S, Dogra M, Katoch D, et al. Safety and outcome of microincision vitreous surgery in uveitis. Ocul Immunol Inflamm (2016) 18:1-10. doi: 10.3109/09273948.2016.1165259

85. Bansal R, Dogra M, Chawla R, Kumar A. Pars plana vitrectomy in uveitis in the era of microincision vitreous surgery. Ind J Ophthalmol (2020) 68:184451. doi: 10.4103/ijo.IJO_1625_20

86. Perumal N, Manicam C, Steinicke M, Funke S, Pfeiffer N, Grus FH. Characterization of the human aqueous humour proteome: A comparison of the genders. PLoS One (2017) 12:e0172481. doi: 10.1371/journal. pone. 0172481

87. Csosz E, Deak E, Kallo G, Csutak A, Tozser J. Diabetic retinopathy: Proteomic approaches to help the differential diagnosis and to understand the underlying molecular mechanisms. J Proteomics (2017) 150:351-8. doi: 10.1016/ j.jprot.2016.06.034

88. Li N, Wang N, Zheng J, Liu XM, Lever OW, Erickson PM, et al. Characterization of human tear proteome using multiple proteomic analysis techniques. J Proteome Res (2005) 4:2052-61. doi: 10.1021/pr0501970

89. de Souza GA, Godoy LM, Mann M. Identification of 491 proteins in the tear fluid proteome reveals a large number of proteases and protease inhibitors. Genome Biol (2006) 7:R72. doi: 10.1186/gb-2006-7-8-r72

Conflict of Interest: The authors declare that the research was conducted in the absence of any commercial or financial relationships that could be construed as a potential conflict of interest.

Copyright (c) 2020 Bansal and Gupta. This is an open-access article distributed under the terms of the Creative Commons Attribution License (CC BY). The use, distribution or reproduction in other forums is permitted, provided the original author(s) and the copyright owner(s) are credited and that the original publication in this journal is cited, in accordance with accepted academic practice. No use, distribution or reproduction is permitted which does not comply with these terms. 